\title{
INTERVIEW
}

For reprint orders, please contact: reprints@futuremedicine.com

\section{Eribulin for the treatment of metastatic breast cancer: a top of the table chemotherapy option?}

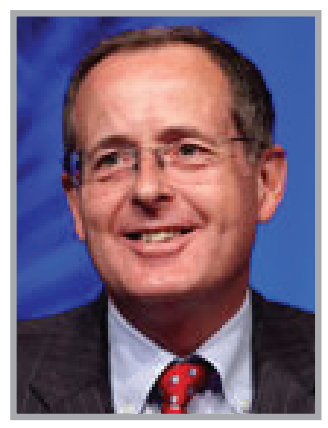

Chris Twelves* speaks to Roshaine Gunawardana, Commissioning Editor: Professor Twelves is Professor of Clinical Cancer Pharmacology and Oncology and heads the Section of Oncology and Cancer Research (Clinical) at the Leeds Institute of Cancer and Pathology (Leeds, UK). He is a medical oncologist with a particular interest in new drug development and clinical pharmacology; his clinical practice has been in colorectal and breast cancer. After training as an oncologist in London he was Senior Lecturer, then Reader, in Medical Oncology in Glasgow at the Beatson Oncology Centre (Glasgow, UK) before taking up his current post at the University of Leeds and Leeds Teaching Hospitals Trust (Leeds, UK). Professor Twelves is Head of the Experimental Cancer Medicine Centre at the Leeds Cancer Research UK Centre (Leeds, UK). He has been a member of the Cancer Research UK New Agents Committee and Chair of the New Drug Development Group of the European Organisation for Reasearch and Treatment of Cancer (EORTC). Professor Twelves has been involved in the development of several important new agents including capecitabine, and eribulin. Professor Twelves has published over 200 papers in journals including the New England Journal of Medicine, Lancet and Journal of Clinical Oncology and spoken at numerous international meetings. He has also edited, or contributed to, several books. He is a past Scientific Chair of the European Cancer Conference.

Q What influenced/led to your interest \& career in medical oncology?

I had always assumed when I was a medical student that I would be a physician working in a hospital rather than becoming a general practitioner or a surgeon. This was not a decision that I had ever consciously made, it was just how I had always assumed it was going to be. I did my general medical training in Sheffield and then worked in London. I was conscious at the time, the early 1980s, that our communication as general physicians talking to patients with cancer was not what it might be; there was not a lot of openness about what was happening to patients and what the diagnosis was. In the instances where doctors were open in telling patients, it was not necessarily done sympathetically. I rapidly realized that when I was talking to cancer patients and telling them that they had cancer, you needed to be able to follow on with some kind of plan about what was going to happen and what the diagnosis meant, rather than just giving them what they effectively might see as a death sentence.

I went into oncology initially just for a few months, to get the experience and skills to be able to talk to patients. Having got into oncology what has continued to appeal has been the variety afforded by the three key elements of treating patients with cancer. First, there is communication with patients. Most of the patients I see have got advanced or metastatic disease, so they are at the relatively late stage of their disease; therefore, I think that communication with them is particularly important. The second

*Section of Oncology \& Clinical Research, Leeds Institute of Molecular Medicine, St. James Institute of Oncology, Leeds, UK; C.J.Twelves@leeds.ac.uk

\section{KEYWORDS}

- breast cancer

- chemotherapy • eribulin 
element is the medical management of treating patients with cancer, treating their symptoms and using chemotherapy drugs or targeted therapies, which often have significant side effects. Finally, we have of course seen many advances and changes resulting from clinical research over the last 20 or 30 years that has made this a very exciting area to be working in.

Q One of your specific interests is in drug development. How have you seen the area of drug development \& research into new treatments in oncology change through your career so far?

My first job as a trainee in oncology was almost 30 years ago, so I have been in oncology for a long time, during which we have seen really very substantial differences, improvements and changes. These have not been what I suppose we, our patients and indeed the media would like to see in terms of overnight breakthroughs. They have tended to be steady, incremental benefits. If I look back at the whole patient experience, including their treatment in 1984, for example, and then compare that in successive decades in 1994, 2004 and now, it is clear that over those four snapshots in time there have been enormous changes in terms of how we communicate with patients, how much they understand and what we tell them about their disease but also in the facilities that we have to treat patients and the use of anticancer drugs. We have many more effective anticancer drugs than we had 30 years ago; radiotherapy and surgery have also changed significantly. In addition to the direct anticancer drugs, we have got a number of other drugs that make a significant difference to treatment, for example, we have much more effective antiemetics than we had when I started in oncology. Similarly, we now have effective treatments for a side effect of breast cancer known as hypercalcemia. So over my time in oncology the transformation has been really quite remarkable.

Q You are the head of the Cancer Medicine
Group \& Experimental Cancer Medicine
Centre in Leeds. Are there any projects
currently ongoing here, on breast cancer
specifically? If so, can you briefly tell us about
them?
I split my time between National Health
Service practice and the university since I am
an employee of the University of Leeds. I spend
half of my research time on patients with breast

cancer treated on clinical trials and the other half is based around what we call early clinical trials. These early trials look at more experimental treatments being tested in patients for the first time, often in a range of different tumor types. It is through that interest in early clinical trials that I have been involved in the development of the oral chemotherapy drug capecitabine and in treating the first patients in the UK to receive that drug right through to its approval and registration as a treatment for breast and bowel cancer. Being involved in the development of new treatments has been very rewarding.

In terms of our own activities in Leeds, we have got a particular interest in immunology and viral therapies. Over last 30 years, I have seen chemotherapy mature in terms of its role as a treatment; although the development of new cytotoxics is not 'fashionable', I think it would be wrong to dismiss the development of new, cytoreductive systemic chemotherapies. Targetted therapies receive much more attention these days, but it may be that, at least in breast cancer, we have probably seen much, if not most of the benefit we are going to get from such drugs. I have been excited by newer approaches, looking at viral therapies and targeting the immune system. I think these approaches potentially represent the next step change in how we are going to continue the move from chemotherapy in the 1970s and 1980s to targeted therapies in the 1990s and the previous decade, to this next decade or so, when immunotherapy and viral therapies offer the prospect of making a substantial difference. Q You are one of the investigators in the
EMBRACE Study \& Study 301 on the use of eribulin mesylate in patients with locally advanced or metastatic breast cancer who had progressed after receiving prior therapy including an anthracycline \& a taxane in either the adjuvant or metastatic setting. Some data were recently presented at the American Society of Clinical Oncology 2014. Can you please explain the main findings of the study? The studies above were conducted with eribulin, which is a novel cytotoxic. Again, I think it is worth emphasizing that, although we are fortunate in having many targeted and hormonal therapies for patients with breast cancer, chemotherapy remains very much at the core of how we treat patients with metastatic disease and there remains the need for new treatments for these 
women. Women with metastatic disease have substantially better quality of life than they did 30 years ago and they undoubtedly have much improved survival. Nevertheless, their survival is limited and metastatic breast cancer remains essentially a disease which we treat with palliative intent. Our patients need more effective drugs of all types that can improve their survival and maintain their quality of life.

Eribulin is an interesting new cytotoxic developed from an extract of a sea sponge identified off the coast of Japan. There has been quite an interest in looking at marine compounds as a source of new cancer treatments as many of the cytotoxics that we currently use are based on plants or other extracts from terrestrial compounds, the best known example probably being paclitaxel. Since the majority of the world is underwater, this seems an attractive place to look for new compounds. Some decades ago, a cytotoxic element of this sponge extract was isolated from the marine sponge Halichondria okadai, a very complex and entirely novel molecule. A synthetic derivative of halichondrin B (eribulin) was developed by Eisai (Tokyo, Japan) and went into early clinical trials where it showed promising activity. Eribulin was then tested in these two large trials in patients with metastatic breast cancer who had already received chemotherapy for their disease.

The first of them to report, EMBRACE (or Study 305) was presented some 3 or 4 years ago now, and conducted in women with heavily pretreated metastatic breast cancer who had already received an anthracyline and a taxane (which are considered the most effective chemotherapy for such women). They were then randomized to receive either the new drug eribulin or whatever the physician and the patient felt would be their normal treatment under these circumstance. When we designed the trial many years ago there was not a single licensed approved drug that would be considered the automatic choice, so rather than constraining the oncologist as to what they would offer women in the control arm, they were able to identify whatever they would normally feel to be their 'standard' single-agent treatment. Within that trial we set the primary end point as improved overall survival; that was quite brave because no trial in this group of women had previously shown improved overall survival. Our feeling was that, given that eribulin was a new cytotoxic that it acted through the microtubule and we already had other agents that interacted, albeit in a different way, with the microtubule, if this was going to be a drug of real interest it had to show a significant and clinically relevant benefit for patients in the form of prolonged survival. And that was what we found, with an increase of on average 2.5 months in the survival of these women from approximately 10.5-13 months. This was something that had not been seen previously in women with such heavily pretreated metastatic breast cancer and the improvement in overall survival was statistically, as well as clinically, significant.

In parallel with that study, although it was not reported until rather later, there was a second trial (Study 301) in women whose cancer was again metastatic, but who had received rather less chemotherapy pretreated than those in the earlier study. In Study 301, the patients were randomized either to eribulin or to the oral chemotherapy drug capecitabine, which would have been considered standard treatment in that situation. It did not satisfy the statistical end point of demonstrating improved overall survival but there was a clear trend in favor of improved overall survival favoring eribulin over capecitabine. In particular, for women with HER2-negative metastatic breast cancer, overall survival was 15.9 months for eribulin versus 13.5 months for capecitabine, and the apparant benefit was even greater for those with triple-negative breast cancer. In certain of the subgroups that we identified and looked at separately, that benefit was statistically significant. Quality of life improved in patients treated with eribulin and those treated with capecitabine. Overall, therefore, the study identified eribulin as being at least as effective as capecitabine, and in certain groups of women, apparently more effective.

Because we now recognize different molecular subgroups of patients, for example, estrogen receptor-positive, progesterone receptor-positive, HER2-positive, HER2-negative, HER2 triple-negative, when we look at the efficacy of a new treatment across each of these molecular subtypes, the number of patients in each of these subgroups is relatively small. Therefore, although we powered the trial statistically to a show a difference between the control arm, and in this case the eribulin-treated arm, overall we do not have the statistical power to look at some of these molecular subgroups; that was one of the reasons for combining these two large studies to give us a total of over 1800 patients and a larger number of patients within each of those molecular subgroups. 
The pooled analysis that we presented at the American Society of Clinical Oncology 2014 showed first of all that the improved overall survival identified in the EMBRACE trial, was maintained in the pooled trial analysis. When we looked at some of the molecular subgroups in more detail it was clear that the patients with HER2-negative disease and patients with triplenegative disease in particular, were getting most benefit from eribulin compared with the control arm in terms of prolonged improvement in their survival.

\section{Q Can you tell us about the mechanism of} action of eribulin mesylate?

Eribulin is a microtubule dynamics inhibitor and the first in this new class of halichondrin. It is similar, but different, to other agents that target the microtubule and I think that there is an important distinction. Some of the other drugs that have being introduced were very much variants on existing drugs; for example, a number of variants on paclitaxel have been developed. In the case of eribulin, however, it is derived from an entirely novel molecule. It acts through the microtubule, which is considered to be very much a 'validated' target in breast cancer, but eribulin interacts with the microtubule in a different way compared with the vinca alkaloids, taxanes and epothilones. So, eribulin is not simply another 'me too' drug, but a genuinely novel molecule. We can make the comparison with targeted therapies where we are quite comfortable having a range of small molecules and monoclonal antibodies that target the HERfamily of receptors; equally we have a number of agents that target the microtubule in a different way, emphasizing that there is novelty with the eribulin.

Q What is the significance of these results in the current climate of breast cancer research \& available treatments?

I think what the two trials have shown individually, and together in the joint analysis, that in the treatment of metastatic breast cancer, eribulin is very much a drug that belongs at the top of the table in terms of chemotherapy options. We have got no shortage of drugs that can be used in treating patients with metastatic breast cancer, and most of us would consider that the best established of these are the anthracyclines and the taxanes, and more recently capecitabine. However, previously, once you went beyond those three, there really were not a lot of data to support the use of the other cytotoxics that we tended to use on an empirical basis. Now, with eribulin, we have a drug that can improve overall survival in patients pretreated with an anthracycline and a taxane and that sort of improvement in overall survival has not been demonstrated for the other drugs that up until recently we were using in these women. So for me, the four key chemotherapy drugs for patients with metastatic breast cancer now includes eribulin. Given that our treatment of patients with metastatic disease is predicated on trying to prolong their life and to maintain their quality of life as long as possible, I think it is important that patients have the opportunity to benefit from the agents of known, proven benefit which we do not have rather than other drugs that we used in the past.

\section{Q What further work needs to be carried out with this drug in order to address patient needs?}

There are a range of other studies that have either commenced or are being planned. There is a large study in women with metastatic disease looking specifically at patients with HER2negative disease, but women who are not as heavily pretreated as in these studies we have been discussing. Eribulin has also been given in combination with trastuzumab; the combination is certainly effective. There are also some early studies looking at the incorporation of eribulin in neoadjuvant or adjuvant therapy principally through the US Cooperative Groups. The move is now to evaluate eribulin earlier in the natural history of the disease.

\section{Q How do you feel about emerging cancer therapeutics: in particular for metastatic breast cancer? Do you think the future is promising?}

I think in breast cancer we were fortunate in that even before the era of recent targeted therapies we already had similar treatments in the form of tamoxifen and other endocrine therapies for women with estrogen receptor-positive disease. Indeed, we now have what I don't think many of us might have expected 30 years ago; that is, new generations of endocrine therapy with fulvestrant and the aromatase inhibitors. And with the addition of everolimus to exemestane, we have for the first time a means of reversing a resistance to endocrine agents. There are, of 
course, other important developments in the area of targeted therapy in patients with HER2positive disease. Trastuzumab itself was a huge advance and that has been followed by lapatinib and more recently pertuzuma. T-DM1, which comprises trastuzumab and a highly active cytotoxic, is particlarly exciting, offering the unusual combination of greater efficacy but less toxicity for the treatment of women with HER2-positive disease.

What we have not seen is specific targeted therapies for patients with triple-negative disease; it is maybe likely that this will prove to be a group where there are a number of specific genetic drivers, each affecting relatively small numbers of patients, so it may be difficult to pin down the targets for those patients. In breast cancer, we already started halfway down the road toward targeted therapy because of our use of endocrine therapies. We may turn out to have not exhausted options for targeted therapy in breast cancer, but to have less far to travel down that road than some other tumor types.

I think the prospect of a step change toward immunotherapies, not in place of the treatments that we have already talked about, but complimenting them, and most likely in combination with these other treatments, is where there is real hope for the future. It is a bit of a cliché these days to use this phrase, but if there is going to be a 'paradigm shift', it is perhaps most likely to be through these novel immune related treatments,
I think, rather than specific new targets in breast cancer.

Q Do you have any closing thoughts for the readers of Breast Cancer Management?

I suppose, only to emphasize, that while on the one hand we have seen major improvements in the treatment of breast cancer, metastatic disease remains a major killer. Breast cancer remains a common disease, and one that is becoming more common so although we now see approximately $75 \%$ of women diagnosed with breast cancer and cured, the remaining 25\%, constitute a large number of women in whom there remains a large, unmet need. It would be wrong for any of us to see breast cancer as something that is 'sorted' and that there is no need for new treatments. That need most certainly remains.

\section{Disclaimer}

The opinions expressed in this interview are those of the interviewee and do not necessarily reflect the views of Future Medicine Ltd.

Financial \& competing interest disclosure

$C$ Twelves is on the speakers bureau for Eisai. C Twelves has no other relevant affiliations or financial involvement with any organization or entity with a financial interest in or financial conflict with the subject matter or materials discussed in the manuscript apart from those disclosed.

No writing assistance was utilized in the production of this manuscript. 